\title{
Discrete-mode cancellation mechanism for high- $Q$ integrated optical cavities with small modal volume
}

\author{
Aristeidis Karalis, Steven G. Johnson, and J. D. Joannopoulos \\ Department of Physics and Center for Material Science and Engineering, Massachusetts Institute of Technology, \\ Cambridge, Massachusetts 02139
}

Received May 4, 2004

\begin{abstract}
A mechanism to reduce radiation loss from integrated optical cavities without a complete photonic bandgap is introduced and demonstrated. It is applicable to any device with a patterned substrate (including both low and high index-contrast systems), when it supports discrete guided or leaky modes through which power escaping the cavity can be channeled into radiation. One then achieves the associated increase in $Q$ by designing the cavity such that the near-field pattern becomes orthogonal to these discrete modes, therefore canceling the coupling of power into them and thus reducing the total radiation loss. The method is independent of any delocalization mechanism and can be used to create high- $Q$ cavities with small modal volume. (C) 2004 Optical Society of America

OCIS codes: $130.3120,230.5750$.
\end{abstract}

A critical component of optical devices is a resonant cavity, characterized by its radiation loss (or lifetime $Q$ ) and its modal volume $V$. Without a complete photonic bandgap ${ }^{1}$ or special symmetries, ${ }^{2}$ cavities normally have intrinsic losses. However, incomplete-gap systems, e.g., those that combine index guiding with one- or two-dimensional bandgaps, ${ }^{3}$ are of widespread interest because they are easily fabricated. Here, we focus on systems in which the periodic pattern extends into the substrate (and the superstrate), with either high or low vertical index contrast, such as those shown in Figs. 1(a) and 1(b). In a uniform substrate (e.g., a bridge ${ }^{4}$ or a membrane ${ }^{5}$ suspended in air), one can achieve a strong peak in $Q$ without modifying $V$ by forcing the cancellation of a multipole term in the radiated far field ${ }^{6}$ or, in a related way, by canceling the dominant Fourier components in the near field ${ }^{5,7-9}$; the key feature, we argue, is a qualitative change in the far-field-Fourier pattern. We show that this cancellation mechanism is substantially modified by a patterned substrate because the radiation is no longer purely a continuum: It can be dominated by discrete guided or leaky modes propagating vertically in the central unpatterned region of the cladding [Fig. 1(c)]. In this case, which applies to arbitrary index-contrast substrates, one forces an orthogonality of the near field with such a discrete mode, resulting in a peak in $Q$. Again, no sacrifice in modal volume $V$ is needed, unlike in previous research, which showed how to increase $Q$ by delocalizing the mode either horizontally ${ }^{10}$ or vertically. ${ }^{11}$ In this Letter, first we describe the general mathematical foundations for all such cancellation mechanisms (with guided, leaky, and continuum modes) and then we give two, two-dimensional example systems (with high and low index contrast) illustrating a factor-of-10 increase in $Q$. Improvement of the same order is expected in three dimensions, as similar gains were demonstrated in three dimensions for multipolebased cancellations. ${ }^{6}$

Consider, as examples, the integrated cavities shown in Figs. 1(a) and 1(b). If the resonant near field does not see the solid substrate and the air superstrate, these can be ignored. This is true if the array of holes is etched deep enough through the high-index slab, such that the holes cover at least the decay length of the waveguide mode. ${ }^{11}$ Labeling the vertical out-of-plane direction $z$, we can now think of the effective patterned cladding as a multilayered waveguide along $z$, which at a resonant frequency $\omega$ supports a complete set of modes [with dependence $\exp (i \beta z-i \omega t)]$ that consists of a continuous spectrum and possibly discrete indexand bandgap-guided modes. Those modes propagating in $z$ (real $\beta$ ) fall inside the radiation cone and thus contribute to the total loss, whereas the evanescent modes (complex $\beta$ ) do not. The mode profiles for the transverse to $z$ field components will be $\mathbf{e}_{n}(x, y)$ and $\mathbf{h}_{n}(x, y)$ for the discrete modes and $\mathbf{e}(\beta ; x, y)$ and $\mathbf{h}(\beta ; x, y)$ for the continuum. Having ignored the solid

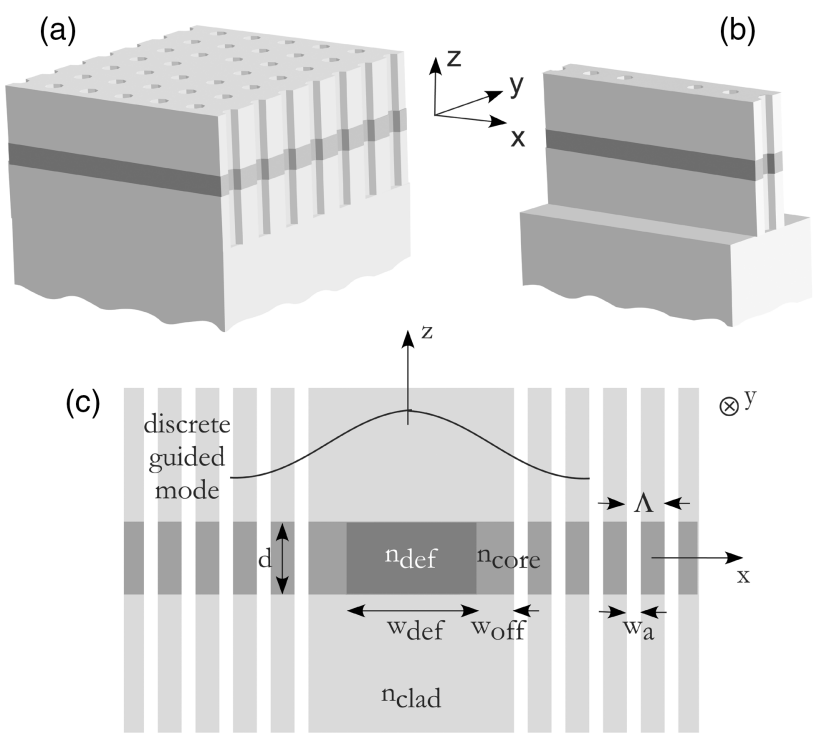

Fig. 1. (a) Photonic-crystal slab microcavity, (b) monorail microcavity, and (c) two-dimensional model of the cavities for the region in the vicinity of the defect. 
substrate and the air superstrate, and dropping the $x, y$ dependence for simplicity, we can write the field outside the cavity as ${ }^{12}$

$$
\begin{aligned}
{\left[\begin{array}{c}
\mathbf{E}_{t}(\mathbf{r}) \\
\mathbf{H}_{t}(\mathbf{r})
\end{array}\right]=} & \sum_{n} c_{n}\left[\begin{array}{c}
\mathbf{e}_{n} \\
\mathbf{h}_{n}
\end{array}\right] \exp \left(i \beta_{n} z\right) \\
& +\int_{\beta} c(\beta)\left[\begin{array}{c}
\mathbf{e}(\beta) \\
\mathbf{h}(\beta)
\end{array}\right] \exp (i \beta z) \mathrm{d} \beta .
\end{aligned}
$$

If this complete set of modes is orthonormalized such that

$$
\begin{gathered}
\frac{1}{4} \int_{x y}\left[\mathbf{e}_{n}^{*} \times \mathbf{h}_{m}+\mathbf{e}_{m} \times \mathbf{h}_{n}^{*}\right] \cdot \hat{\mathbf{z}} \mathrm{d} x \mathrm{~d} y=\delta_{m n}, \\
1 / 4 \int_{x y}\left[\mathbf{e}(\beta) \times \mathbf{h}\left(\beta^{\prime}\right)+\mathbf{e}\left(\beta^{\prime}\right) \times \mathbf{h}^{*}(\beta)\right] \\
\cdot \hat{\mathbf{z}} \mathrm{d} x \mathrm{~d} y=\delta\left(\beta-\beta^{\prime}\right)
\end{gathered}
$$

the total power radiated will be (for any $z$ )

$$
\begin{aligned}
P & =\frac{1}{2} \operatorname{Re}\left[\int_{x y} \mathbf{E}_{t} \times \mathbf{H}_{t}^{*} \cdot \hat{\mathbf{z}} \mathrm{d} x \mathrm{~d} y\right] \\
& =\sum_{n: \beta_{n} \text { real }}\left|c_{n}\right|^{2}+\int_{\beta \text { real }}|c(\beta)|^{2} \mathrm{~d} \beta,
\end{aligned}
$$

namely, an incoherent sum of discrete terms and a continuum. If the stored energy inside the cavity is $U=\int u \mathrm{~d} V$, and $u=1 / 2 \epsilon|\mathbf{E}|^{2}$ is the energy density, then the two major figures of merit that characterize a resonant cavity are quality factor $Q$ (Ref. 13) and modal volume $V$ (Ref. 14):

$$
\begin{aligned}
Q^{-1} & =\frac{P}{\omega U}=\sum_{n: \beta_{n} \text { real }} Q_{n}{ }^{-1}+\int_{\beta \text { real }} Q^{-1}(\beta) \mathrm{d} \beta \\
V & =U / \max (u),
\end{aligned}
$$

where the introduced individual factors $Q_{n}{ }^{-1}=$ $\left|c_{n}\right|^{2} / \omega U$ and $Q^{-1}(\beta)=|c(\beta)|^{2} / \omega U$ contribute independently to the total $Q$. For a low-loss cavity, $Q$ must be large, whereas a small modal volume is usually desired.

Depending on the design, the discrete terms in Eq. (3) may often carry a significant portion of the total radiated power, so $Q$ is limited by some $Q_{n}$, as shown below. In that case, one can achieve a significant increase in $Q$ by canceling the corresponding expansion coefficients. These are determined by the near-field pattern $\mathbf{E}_{t 0}(x, y)=\mathbf{E}_{t}(x, y, z=0)$ and $\mathbf{H}_{t 0}(x, y)=\mathbf{H}_{t}(x, y, z=0)$ :

$$
\begin{aligned}
{\left[\begin{array}{c}
c_{n} \\
c(\beta)
\end{array}\right]=} & \frac{1}{4} \int_{x y}\left\{\left[\begin{array}{c}
\mathbf{e}_{n} \\
\mathbf{e}(\beta)
\end{array}\right]^{*} \times \mathbf{H}_{t 0}+\mathbf{E}_{t 0} \times\left[\begin{array}{c}
\mathbf{h}_{n} \\
\mathbf{h}(\beta)
\end{array}\right]^{*}\right\} \\
& \times \hat{\mathbf{z}} \mathrm{d} x \mathrm{~d} y .
\end{aligned}
$$

One can achieve cancellation of $c_{n}$ by forcing the near field to be orthogonal to its associated mode by proper design of the defect, for example, by tuning its dielectric constant or shape. Similarly, elimination of a mode $c(\beta)$ within the continuum can be imposed again through Eq. (6). In any case, cancellation of an eigenmode of this generalized Fourier-type basis set for the near field implies, according to Fraunhofer diffraction theory, a corresponding nodal direction in the far field.

The multilayered waveguide formed by the patterned cladding might also support discrete modes of the leaky type. Their contribution to the radiated field associated with the continuum can be made evident by application of a steepest-descent approximation to Eq. (1) for some angle of observation. If then leaky wave poles $\tilde{\beta}_{m}$ are crossed during the deformation of the integration path in the complex $\beta$ plane, Eq. (1) becomes ${ }^{15}$

$$
\begin{aligned}
{\left[\begin{array}{l}
\mathbf{E}_{t}(\mathbf{r}) \\
\mathbf{H}_{t}(\mathbf{r})
\end{array}\right] \approx } & \sum_{n} c_{n}\left[\begin{array}{c}
\mathbf{e}_{n} \\
\mathbf{h}_{n}
\end{array}\right] \exp \left(i \beta_{n} z\right) \\
& +\sum_{m} \tilde{c}_{m}\left[\begin{array}{c}
\tilde{\mathbf{e}}_{m} \\
\tilde{\mathbf{h}}_{m}
\end{array}\right] \exp \left(i \tilde{\beta}_{m} z\right)+\int_{\mathrm{SD} \mathrm{path}}[] \mathrm{d} \beta .
\end{aligned}
$$

Inasmuch as leaky modes do not belong to a complete orthogonal set, relations of the form of Eq. (3) or (4) cannot be rigorously written. Still, canceling a discrete coefficient $\tilde{c}_{m}$ in expression (7) should result in an increase of radiation $Q$ if the associated leaky wave carries a significant portion of the field radiated within the continuum.

Just like the free-space cancellations, ${ }^{6}$ such a discrete-mode cancellation involves only small changes in the near-field pattern to force the orthogonality relation, and it does not rely on delocalization. Modal volume can thus be kept small and nearly constant.

To illustrate the principles above, we used the simplified two-dimensional model of Fig. 1(c) to describe the region in the vicinity of the cavities in Figs. 1(a)
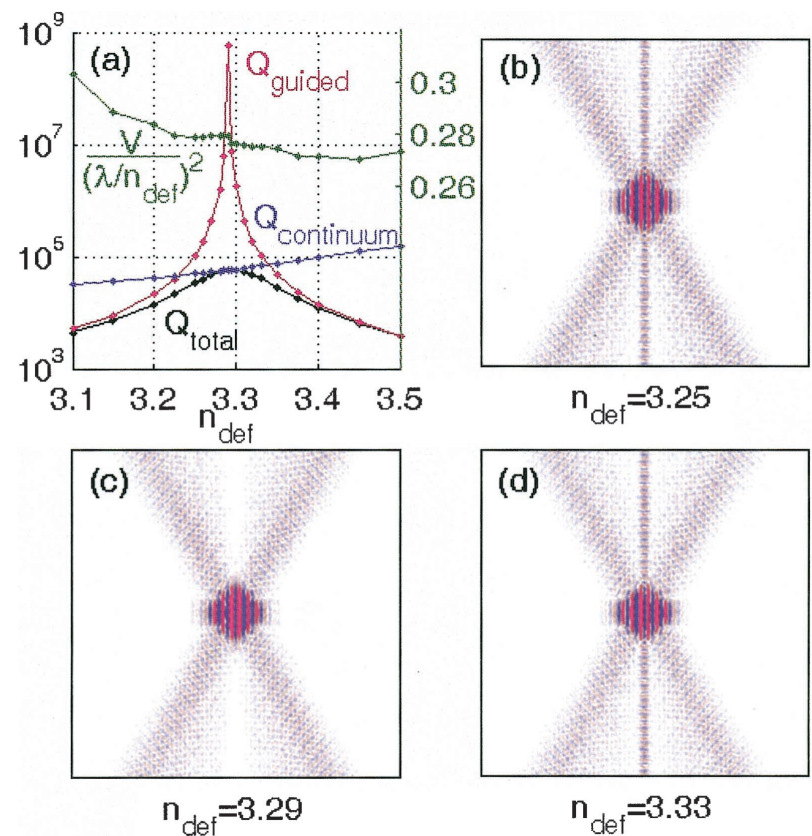

Fig. 2. Results for $n_{\text {core }}=3.35, n_{\text {clad }}=3.17, d=680 \mathrm{~nm}$, $\Lambda=320 \mathrm{~nm}, w_{a}=150 \mathrm{~nm}$, and $w_{\text {off }}=125 \mathrm{~nm}$. (a) $Q$ total, $Q$ of the guided mode, $Q$ of the continuum, and $V /\left(\lambda / n_{\text {def }}\right)^{2}$ versus $n_{\text {def }}\left(w_{\text {def }}\right.$ is adjusted such that $\lambda=$ $1.55 \mu \mathrm{m})$; (b) -(d) resonant field at three values of $n_{\text {def }}$. 

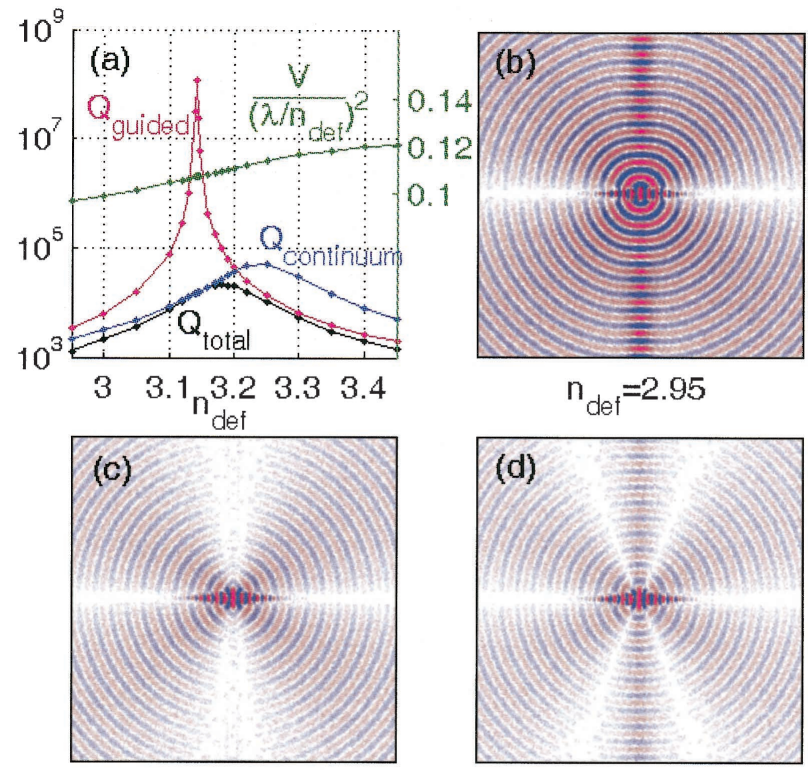

$\mathrm{n}_{\text {def }}=3.142$

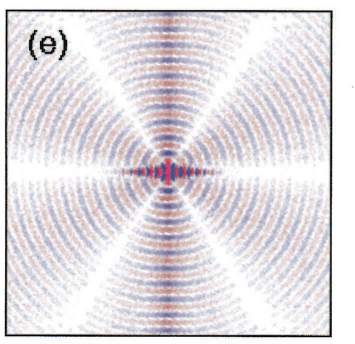

$\mathrm{n}_{\text {def }}=3.221$

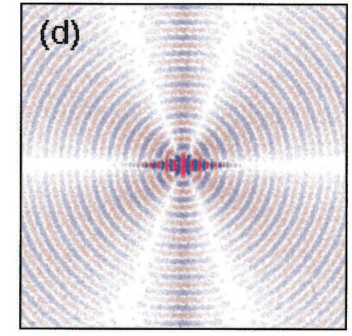

$\mathrm{n}_{\mathrm{def}}=3.18$

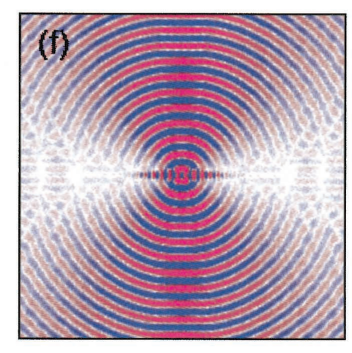

$\mathrm{n}_{\text {def }}=3.45$

Fig. 3. Results for $n_{\text {core }}=3.45, n_{\text {clad }}=1.45, d=1 \mu \mathrm{m}$, $\Lambda=300 \mathrm{~nm}, w_{a}=100 \mathrm{~nm}$, and $w_{\text {off }}=100 \mathrm{~nm}$. (a) $Q$ total, $Q$ of the guided mode, $Q$ of the continuum, and $V /\left(\lambda / n_{\text {def }}\right)^{2}$ versus $n_{\text {def }}$; (b)-(f) resonant field at five values of $n_{\text {def }}$.

and 1(b). TE ( $y$-polarized) modes were examined by an eigenmode expansion method. ${ }^{16}$ A periodic array of deep air grooves is etched all the way through a single-mode symmetric slab waveguide, opening a bandgap in the $x$ direction. We then introduce a defect by making one waveguide section longer. The resonant field will leak power vertically out of the cavity. To study the effects of defect variations on radiation loss, we varied the index $n_{\text {def }}$ of a part of the waveguide core [Fig. 1(c)] while simultaneously adjusting its length $w_{\text {def }}$, to keep the resonant wavelength fixed; maintaining a fixed position in the gap is critical to preserving the localization and not confusing the cancellation and delocalization mechanisms for improving $Q$.

For a low index-contrast InP/GaInAsP $(n=3.35-$ 3.17) waveguide with a bandgap in the 1.205$1.949-\mu \mathrm{m}$ range, a typical field plot for the secondorder (two nodes in the defect) resonance at $\lambda=$ $1.55 \mu \mathrm{m}$ is shown in Fig. 2(b). The majority of the radiation loss is index confined in the central region to a discrete, vertically propagating guided mode, whereas some of the extended modes $\mathbf{e}(\beta ; x) \exp (i \beta z)$ have been removed by the bandgap. For $n_{\text {def }}=3.29$ the guided mode is canceled [Figs. 2(a) and 2(b)]

with a corresponding factor-of-10 increase in total $Q$, and the peak is limited only by the continuum to $Q \approx 58,000$. Moreover, the modal volume is almost independent of the tuning and is very small, of the order of $0.28\left(\lambda / n_{\text {def }}\right)^{2}$ [Fig. 2(a)].

A high-index-contrast $\mathrm{Si} / \mathrm{SiO}_{2}(n=3.45-1.45)$ waveguide with a $1.205-1.651-\mu \mathrm{m}$ bandgap has, for the same resonance at $\lambda=1.55 \mu \mathrm{m}$, a field pattern as in Fig. 3(b). Because of the smaller cladding index variation $\left(\right.$ air $-\mathrm{SiO}_{2}$ ) the discrete guided mode is now less confined, and its contribution to radiated power is no longer dominant but is similar to that of the continuum. Two equally prominent cancellations are now present: One of the discrete eigenmode types presented in this Letter, at $n_{\text {def }}=3.142$ [Figs. 3(a) and $3(\mathrm{c})]$, and one of the multiple types ${ }^{6}$ at $n_{\text {def }}=3.221$ [Figs. 3(a) and 3(e)]. The total $Q$ is maximized to $Q \approx$ 21,000 midway, at $n_{\text {def }}=3.18$ [Figs. 3(a) and 3(d)]. The null in the far-field pattern is sweeping in angle from the vertical $z$ to the horizontal $x$ directions as tuning parameter $n_{\text {def }}$ is varied and a different discrete or extended mode is canceled. The modal volume is again roughly constant and is even smaller than before at $0.11\left(\lambda / n_{\text {def }}\right)^{2}$ [Fig. 3(a)].

This study was supported by the Materials Research Science and Engineering Center program of the National Science Foundation under award DMR-9400334. A. Karalis's e-mail address is aristos@mit.edu.

\section{References}

1. J. D. Joannopoulos, R. D. Meade, and J. N. Winn, Photonic Crystals: Molding the Flow of Light (Princeton U. Press, Princeton, N.J., 1995).

2. M. R. Watts, S. G. Johnson, H. A. Haus, and J. D. Joannopoulos, Opt. Lett. 27, 1785 (2002).

3. S. G. Johnson and J. D. Joannopoulos, Photonic Crystals: The Road from Theory to Practice (Kluwer Academic, Boston, Mass., 2002).

4. P. R. Villeneuve, S. Fan, J. D. Joannopoulos, K.-Y. Lim, G. S. Petrich, L. A. Kolodziejski, and R. Reif, Appl. Phys. Lett. 67, 167 (1995).

5. J. Vučković, M. Lončar, H. Mabuchi, and A. Scherer, Phys. Rev. E 65, 016608 (2002).

6. S. G. Johnson, S. Fan, A. Mekis, and J. D. Joannopoulos, Appl. Phys. Lett. 78, 3388 (2001).

7. K. Srinivasan and O. Painter, Opt. Express 11, 579 (2003), http://www.opticsexpress.org.

8. H.-Y. Ryu, M. Notomi, and Y.-H. Lee, Appl. Phys. Lett. 83, 4294 (2003).

9. Y. Akahane, T. Asano, B.-S. Song, and S. Noda, Nature 425, 944 (2003).

10. S. G. Johnson, A. Mekis, S. Fan, and J. D. Joannopoulos, Comput. Sci. Eng. 3, 38 (2001).

11. H. Benisty, P. Lalanne, S. Olivier, M. Rattier, C. Weisbuch, C. J. M. Smith, T. F. Krauss, C. Jouanin, and D. Cassagne, Opt. Quantum Electron. 34, 205 (2002).

12. D. Marcuse, Theory of Dielectric Optical Waveguides, 2nd ed. (Academic, San Diego, Calif., 1991).

13. H. A. Haus, Waves and Fields in Optoelectronics (Prentice-Hall, Englewood Cliffs, N.J., 1984).

14. E. M. Purcell, Phys. Rev. B 69, 681 (1946).

15. T. Tamir and A. A. Oliner, Proc. IEE 110, 310 (1963).

16. P. Bienstman and R. Baets, Opt. Quantum Electron. 33, 327 (2001). 